QUARTERLY OF APPLIED MATHEMATICS

VOLUME LXVIII, NUMBER 4

DECEMBER 2010, PAGES 661-669

S 0033-569X(2010)01184-8

Article electronically published on September 3, 2010

\title{
A NOTE ON YOUNG MEASURES AND CORRECTORS IN $\Gamma$-CONVERGENCE AND HOMOGENIZATION
}

\author{
$\mathrm{BY}$ \\ PABLO PEDREGAL \\ Departamento de Matemáticas, ETSI Industriales, Universidad de Castilla-La Mancha, 13071 Ciudad \\ Real, Spain
}

Abstract. We explore a general strategy to determine the Young measure associated with sequences of pairs of conductivity coefficients and corresponding gradient fields. We also relate this issue to corrector results and investigate various examples.

1. Introduction. Our goal in this note is to determine, under suitable conditions and assumptions, the joint (full) Young measure (2, 14) associated with pairs of sequences $\left\{\left(a_{j}, \nabla u_{j}\right)\right\}$ where there is an intimate relationship between those two sequences of functions to the point that, in typical situations, $u_{j}$ is determined in a unique way from $a_{j}$. The simplest situation where we can explain our objective comes directly from homogenization. Suppose that given $\left\{a_{j}\right\}$, a sequence of scalar functions bounded uniformly away from zero in $\Omega \subset \mathbf{R}^{N}$, we solve the conductivity equation

$$
\operatorname{div}\left[a_{j}(x) \nabla u_{j}(x)\right]=0 \text { in } \Omega, \quad u_{j}=u_{0} \text { on } \partial \Omega,
$$

where $u_{j} \in H^{1}(\Omega), u_{j}-u_{0} \in H_{0}^{1}(\Omega)$. We know that $u_{j} \rightarrow u$ in $H^{1}(\Omega)$ where $u$ is the solution of the homogenized problem

$$
\operatorname{div}[A(x) \nabla u(x)]=0 \text { in } \Omega, \quad u=u_{0} \text { on } \partial \Omega,
$$

and the matrix $A$ is appropriately determined from the sequence $\left\{a_{j}\right\}$ ( $A$ is the H-limit of $\left.\left\{a_{j}\right\}[8]\right)$. We might be interested in knowing the limit behavior of certain physical quantities of interest which can be easily computed for each problem $j$. One such example is the dissipated heat ([7]), if we think in terms of electric conductivity, given by the integral

$$
H_{j}=\int_{\Omega} \frac{1}{a_{j}(x)}\left|\nabla u_{j}(x)\right|^{2} d x .
$$

The issue we would like to address is how to find the limit of such quantities as $j \rightarrow \infty$, so that we can say what the "true" dissipated heat is for the limit homogenized problem.

Received February 13, 2009.

2010 Mathematics Subject Classification. Primary 35B27, 49J45, 74Q05.

Supported by project MTM2007-62945 (MEC, Spain) and by project PCI08-0084-0424 from JCCM (Castilla-La Mancha).

E-mail address: pablo.pedregal@uclm.es 
In fact, our objective is much more ambitious. We pretend to give a rule to calculate the limit of integrals of the form

$$
\int_{\Omega} F\left(x, a_{j}(x), \nabla u_{j}(x)\right) d x
$$

for arbitrary Carathéodory integrands $F(x, \lambda, \rho)$ (continuous in $(\lambda, \rho)$ and measurable in $x$ ), provided that such compositions $\left\{F\left(x, a_{j}(x), \nabla u_{j}(x)\right)\right\}$ make up an equiintegrable family of functions in $L^{1}(\Omega)$. This issue is nothing but finding the Young measure corresponding to the sequence (or an appropriate subsequence) of pairs $\left\{\left(a_{j}, \nabla u_{j}\right)\right\}$.

In an equivalent format in the context of $\Gamma$-convergence of functionals ([5]), we may consider the sequence of quadratic functionals

$$
I_{j}(v)=\int_{\Omega} \frac{a_{j}(x)}{2}|\nabla v(x)|^{2} d x .
$$

We know that the $\Gamma$-limit is given by the functional

$$
I(v)=\int_{\Omega} \frac{1}{2} \nabla v(x)^{T} A(x) \nabla v(x) d x
$$

for an appropriate matrix field $A(x)$. Suppose that for any given $u \in H^{1}(\Omega)$, not just the solution of the homogenized problem, we have a sequence $\left\{u_{j}\right\}$ such that $u_{j} \rightarrow u$ in $H^{1}(\Omega)$ and

$$
\lim _{j \rightarrow \infty} I_{j}\left(u_{j}\right)=I(u)
$$

Can we say something about the limits

$$
\lim _{j \rightarrow \infty} \int_{\Omega} F\left(x, a_{j}(x), \nabla u_{j}(x)\right) d x
$$

for an arbitrary Carathéodory integrand $F$ as before? That is, can we determine the full Young measure corresponding to the sequence of pairs $\left\{\left(a_{j}, \nabla u_{j}\right)\right\}$ ? Notice that this question is a bit more general than the one considered above in the language of homogenization, as the sequence $\left\{u_{j}\right\}$ does not have to be the sequence of minimizers for $I_{j}$.

As a matter of fact, we would like to place ourselves in a more general context in which non-linear problems may eventually be examined. We will consider, dwelling in the context of $\Gamma$-convergence, a sequence of functionals of the type

$$
I_{j}(v)=\int_{\Omega} W\left(a_{j}(x), \nabla v(x)\right) d x
$$

under suitable assumptions for the integrands $W$. The $\Gamma$-limit of such functionals has already been addressed in some previous papers ([11, [12]), and given in the form of an integral functional

$$
I(v)=\int_{\Omega} \psi(x, \nabla v(x)) d x,
$$

where the integrand $\psi$ is given in a precise way from $W$ and the sequence $a_{j}$. Here we would like to deepen that analysis to determine the full Young measure corresponding to pairs $\left\{\left(a_{j}, \nabla u_{j}\right)\right\}$ under the main hypotheses that $u_{j} \rightarrow u$ and $I_{j}\left(u_{j}\right) \rightarrow I(u)$.

The assumptions on $W(\lambda, \rho): \mathbf{R}^{m} \times \mathbf{R}^{N} \rightarrow \mathbf{R}$ are: 
- growth of order $p>1$ on $\rho$ :

$$
c\left(|\rho|^{p}-1\right) \leq W(\lambda, \rho) \leq C\left(|\rho|^{p}-1\right), \quad 0<c<C ;
$$

- uniform continuity with respect to $\lambda$ :

$$
\left|W\left(\lambda_{1}, \rho\right)-W\left(\lambda_{2}, \rho\right)\right| \leq w\left(\left|\lambda_{1}-\lambda_{2}\right|\right)|\rho|^{p},
$$

where $w$ is continuous and $w(0)=0$;

- strict convexity of $W$ on $\rho$ for every $\lambda$;

- $a_{j}: \Omega \rightarrow \mathbf{R}^{m}$ is a sequence of vector- (matrix-) valued functions which we assume, for simplicity, is uniformly bounded in $L^{\infty}\left(\Omega ; \mathbf{R}^{m}\right)$.

Further to this last requirement on $\left\{a_{j}\right\}$, we mainly assume the AGP (average gradient property) condition. This is a condition specifically designed so that $\Gamma$-limits can be computed or precisely determined. The main example corresponds to the periodic setting. See [11] and [12] for more information. We can say that such a sequence $\left\{a_{j}\right\}$ verifies the AGP condition if, loosely speaking, "averages of gradients over sets where the sequence is essentially constant are gradients themselves". The precise definition is a bit involved and can be found in [12. To avoid unnecessary repetition, we do not include it here as it is not a main issue in this work. All we need to know for our purpose here is that this assumption guarantees that $\Gamma$-limits in the gradient case can be computed through the identity

$$
\begin{gathered}
\psi(x, \rho)=\inf _{\phi}\left\{\int_{\mathbf{R}^{m}} W(\lambda, \phi(\lambda)) d \sigma_{x}(\lambda): \rho=\int_{\mathbf{R}^{m}} \phi(\lambda) d \sigma_{x}(\lambda),\right. \text { and } \\
\text { whenever } r_{j} \searrow 0 \text { is such that }\left\{a_{j}\left(x+r_{j} y\right)\right\} \text { generates } \sigma_{x}, \\
\text { then } \left.\left\|\operatorname{curl}\left(\phi\left(a_{j}\left(x+r_{j} y\right)\right)\right)\right\|_{W^{-1, q}(B)} \rightarrow 0\right\},
\end{gathered}
$$

where $B$ is the unit ball in $\mathbf{R}^{N}$, the family of probability measures $\sigma=\left\{\sigma_{x}\right\}_{x \in \Omega}$ is the Young measure associated with $\left\{a_{j}\right\}$, and $q$ is the dual exponent of $p$ (see Lemma 4.2 of [11]).

Our main results follow.

TheOREM 1. Under the preceding assumptions on the sequence $\left\{a_{j}\right\}$ and the integrand $W(\lambda, \rho)$, for a.e. $x \in \Omega$, every $u \in W^{1, p}(\Omega)$ such that

$$
\int_{\Omega} \psi(y, \nabla u(y)) d y<+\infty
$$

and every sequence $\left\{u_{j}\right\}$ such that $u_{j} \rightarrow u$ and $I_{j}\left(u_{j}\right) \rightarrow I(u)$, we have:

(1) $\left\{\left|\nabla u_{j}\right|^{p}\right\}$ is equiintegrable;

(2) there exists a field $\varphi(\lambda, x)$ such that for a.e. $x \in \Omega, \varphi(\cdot, x) \in L^{1}\left(\sigma_{x}\right)$ and

$$
\nabla u(x)=\int_{\mathbf{R}^{m}} \varphi(\lambda, x) d \sigma_{x}(\lambda), \quad \psi(x, \nabla u(x))=\int_{\mathbf{R}^{m}} W(\lambda, \varphi(\lambda, x)) d \sigma_{x}(\lambda) ;
$$

(3) for every Carathéodory integrand $F$

$$
\lim _{j \rightarrow \infty} \int_{\Omega} F\left(x, a_{j}(x), \nabla u_{j}(x)\right) d x=\int_{\Omega} \int_{\mathbf{R}^{m}} F(x, \lambda, \varphi(\lambda, x)) d \sigma_{x}(\lambda) d x,
$$

provided that $\left\{F\left(x, a_{j}(x), \nabla u_{j}(x)\right)\right\}$ is equiintegrable. 
Notice that this result can be equivalently stated by saying that the Young measure associated with pairs $\left\{\left(a_{j}, \nabla u_{j}\right)\right\}$ is given by

$$
\delta_{\varphi(\lambda, x)} \otimes \sigma_{x}
$$

When the sequence $\left\{u_{j}\right\}$ is precisely the sequence of minimizers for $I_{j}$, then the determination of the Young measure can be understood through corrector results in homogenization (44). Indeed, a corrector result is, at first sight, more than determining the Young measure, though we will see that, at least in our framework, it is equivalent. In our scenario in $\Gamma$-convergence, we will prove a more general result.

Theorem 2. Let $U_{j}: \Omega \subset \mathbf{R}^{N} \rightarrow \mathbf{R}^{N}$ be a sequence of fields (not necessarily gradients) such that $\left\{\left|U_{j}\right|^{p}\right\}$ is equiintegrable, and the Young measure associated with pairs $\left\{\left(a_{j}, U_{j}\right)\right\}$ is given by (11) for a certain Carathéodory field $\varphi: \mathbf{R}^{m} \times \Omega \rightarrow \mathbf{R}^{N}$ (as in the preceding theorem). Then

$$
U_{j}-\varphi\left(a_{j}(x), x\right) \rightarrow 0 \text { strong in } L^{p}(\Omega) .
$$

As an immediate corollary we get the following.

Corollary 3. Suppose that $u \in W^{1, p}(\Omega)$ is such that

$$
\int_{\Omega} \psi(x, \nabla u(x)) d x<+\infty,
$$

and $\left\{u_{j}\right\}$ is as in Theorem 1 with the field $\varphi$, a Carathéodory integrand. Then

$$
\nabla u_{j}(x)-\varphi\left(a_{j}(x), x\right) \rightarrow 0 \text { strong in } L^{p}(\Omega),
$$

so that $\left\{\varphi\left(a_{j}(x), x\right)\right\}$ are the correctors.

Whenever the field $\varphi(\lambda, x)$ can be explicitly computed, we will have the corresponding sequence of correctors. As far as we can tell, there are not many such situations. See [6], [9].

Our objective in this note is to provide an alternative point of view on some issues in homogenization that may be helpful in some situations. We explore the most typical examples in Section 3. The proofs of our main results are described in Section 2.

2. Proofs. Suppose that the sequence of pairs $\left\{\left(a_{j}, \nabla u_{j}\right)\right\}$ generate the joint Young measure

$$
\Lambda=\left\{\Lambda_{x}\right\}_{x \in \Omega}, \quad \Lambda_{x}=\mu_{\lambda, x}(\rho) \otimes \sigma_{x}(\lambda),
$$

where we are using the slicing decomposition onto the Young measure corresponding to $\left\{a_{j}\right\} .\left\{u_{j}\right\}$ converges weakly in $W^{1, p}(\Omega)$ to a certain $u$. The representation in terms of Young measures always yields something smaller (Theorem 6.11 in [10]), so that

$$
\lim _{j \rightarrow \infty} \int_{\Omega} W\left(a_{j}(x), \nabla u_{j}(x)\right) d x \geq \int_{\Omega} \int_{\mathbf{R}^{m}} \int_{\mathbf{R}^{N}} W(\lambda, \rho) d \mu_{\lambda, x}(\rho) d \sigma_{x}(\lambda) d x .
$$


By using Jensen's inequality, we can write

$$
\begin{array}{r}
\int_{\Omega} \int_{\mathbf{R}^{m}} \int_{\mathbf{R}^{N}} W(\lambda, \rho) d \mu_{\lambda, x}(\rho) d \sigma_{x}(\lambda) d x \\
\geq \int_{\Omega} \int_{\mathbf{R}^{m}} W\left(\lambda, \int_{\mathbf{R}^{N}} \rho d \mu_{\lambda, x}(\rho)\right) d \sigma_{x}(\lambda) d x .
\end{array}
$$

We know from [1] that the $\Gamma$-limit is an infimum over fields of the form

$$
\int_{\mathbf{R}^{N}} \rho d \mu_{\lambda, x}(\rho)
$$

coming from all feasible sequences $\left\{\nabla v_{j}\right\}$ having the weak limit $\nabla u$. In particular, we definitely have

$$
\int_{\Omega} \int_{\mathbf{R}^{m}} W\left(\lambda, \int_{\mathbf{R}^{N}} \rho d \mu_{\lambda, x}(\rho)\right) d \sigma_{x}(\lambda) d x \geq \int_{\Omega} \psi(x, \nabla u(x)) d x
$$

since the right-hand side is the minimum over expressions of the form occurring on the left-hand side. By hypothesis, the starting and final points of all these inequalities,

$$
\lim _{j \rightarrow \infty} \int_{\Omega} W\left(a_{j}(x), \nabla u_{j}(x)\right) d x=\int_{\Omega} \psi(x, \nabla u(x)) d x,
$$

are in fact equal. Hence all the intermediate inequalities are, indeed, equalities. In particular, the equality

$$
\lim _{j \rightarrow \infty} \int_{\Omega} W\left(a_{j}(x), \nabla u_{j}(x)\right) d x=\int_{\Omega} \int_{\mathbf{R}^{m}} \int_{\mathbf{R}^{N}} W(\lambda, \rho) d \mu_{\lambda, x}(\rho) d \sigma_{x}(\lambda) d x
$$

implies that $\left\{\left(a_{j}, \nabla u_{j}\right)\right\}$ is equiintegrable because the family of probability measures on the right-hand side is the Young measure associated with the sequence on the left, and due to the growth assumed on $W$ with respect to $\rho$, the sequence $\left\{\left|\nabla u_{j}\right|^{p}\right\}$ is equiintegrable ([10]).

By the strict convexity of $W$ with respect to the same gradient variable $\rho$, we conclude that

$$
\mu_{\lambda, x}=\delta_{\varphi(\lambda, x)}
$$

and, thus, the Young measure corresponding to $\left\{\left(a_{j}, \nabla u_{j}\right)\right\}$ is

$$
\Lambda_{x}=\delta_{\varphi(\lambda, x)} \otimes \sigma_{x}
$$

for a certain field $\varphi(\lambda, x)$ such that $\varphi(\cdot, x) \in L^{1}\left(\sigma_{x}\right)$ for a.e. $x \in \Omega$. Finally, this optimal field should be such that

$$
\nabla u(x)=\int_{\mathbf{R}^{m}} \varphi(\lambda, x) d \sigma_{x}(\lambda), \quad \psi(x, \nabla u(x))=\int_{\mathbf{R}^{m}} W(\lambda, \varphi(\lambda, x)) d \sigma_{x}(\lambda) .
$$

This proves the theorem.

For the proof of Theorem 2, under our assumptions, the sequence of fields

$$
U_{j}(x)-\varphi\left(a_{j}(x), x\right)
$$

is such that its $p$-th power is equiintegrable. Recall that the sequence $\left\{a_{j}\right\}$ is assumed to be uniformly bounded in $L^{\infty}(\Omega)$. Let us examine its associated Young measure. To this 
end, let $G(\lambda)$ be any smooth and bounded integrand. By assumption, because $\delta_{\varphi(\lambda, x)} \otimes \sigma_{x}$ is the Young measure associated with $\left\{\left(a_{j}, U_{j}\right)\right\}$ and $\varphi$ is a Carathéodory integrand,

$$
\lim _{j \rightarrow \infty} \int_{E} G\left(U_{j}(x)-\varphi\left(a_{j}(x), x\right)\right) d x=\int_{E} \int_{\mathbf{R}^{m}} G(\varphi(\lambda, x)-\varphi(\lambda, x)) d \sigma_{x}(\lambda) d x=G(0)|E|
$$

for arbitrary measurable $E \subset \Omega$. The arbitrariness of $G$ and $E$ yields that the Young measure for $\left\{U_{j}(x)-\varphi\left(a_{j}(x), x\right)\right\}$ is $\delta_{0}$. It is well known (see [10 for instance) that this implies the strong convergence claimed in the statement of the Corollary.

3. Examples. We focus on the typical periodic case

$$
I_{j}(v)=\int_{Q} \frac{a_{j}(x)}{2}|\nabla v(x)|^{2} d x, \quad a_{j}(x)=a(j x), x \in Q=(0,1)^{N},
$$

where $a$ is bounded, bounded away from zero, and $Q$-periodic. Define the density

$$
\psi: \mathbf{R}^{N} \rightarrow \mathbf{R}, \quad \psi(\rho)=\int_{Q} \frac{a(\lambda)}{2}\left|\rho+\nabla_{\lambda} \xi(\lambda, \rho)\right|^{2} d \lambda,
$$

where

$$
\xi(\lambda, \rho): \mathbf{R}^{N} \times \mathbf{R}^{N} \rightarrow \mathbf{R},
$$

for each fixed $\rho$, is the unique $Q$-periodic, weak solution in $H^{1}(Q)$ of the cell problem

$$
\operatorname{div}\left[a(\lambda)\left(\rho+\nabla_{\lambda} \xi(\lambda, \rho)\right)\right]=0, \int_{Q} \xi(\lambda, \rho) d \lambda=0 .
$$

The $\Gamma$-limit of $\left\{I_{j}\right\}$ is

$$
I(u)=\int_{\Omega} \psi(\nabla u(x)) d x .
$$

Observe that $\sigma_{x}=d \lambda$, the Lebesgue measure, for a.e. $x \in Q$. 11 It is easy to check ([12]) that the field $\varphi(\lambda, x)$ in Theorem 1 is

$$
\varphi(\lambda, x)=\nabla u(x)+\nabla_{\lambda} \xi(\lambda, \nabla u(x)) .
$$

A direct application of Theorem 1 yields that if $\left\{u_{j}\right\}$ is such that

$$
u_{j} \rightarrow u \text { in } H^{1}(Q), \quad \int_{Q} \frac{a_{j}(x)}{2}\left|\nabla u_{j}(x)\right|^{2} d x \rightarrow \int_{Q} \psi(\nabla u(x)) d x,
$$

then the joint Young measure associated with pairs $\left\{\left(a(j x), \nabla u_{j}(x)\right)\right\}$ is given by

$$
\delta_{\nabla u(x)+\nabla_{\lambda} \xi(\lambda, \nabla u(x))} \otimes d \lambda
$$

that is to say

$$
\int_{Q} F\left(x, a(j x), \nabla u_{j}(x)\right) d x \rightarrow \int_{Q} \int_{Q} F\left(x, a(\lambda), \nabla u(x)+\nabla_{\lambda} \xi(\lambda, \nabla u(x))\right) d \lambda d x
$$

for any Carathéodory integrand $F$ such that the sequence $\left\{F\left(x, a(j x), \nabla u_{j}(x)\right)\right\}$ is equiintegrable in $L^{1}(Q)$.

As remarked above, in the particular case in which

$$
\operatorname{div}\left[a(j x) \nabla u_{j}(x)\right]=0 \text { in } Q, \quad u=u_{0} \text { on } \partial \Omega,
$$

\footnotetext{
${ }^{1}$ There is a typical abuse of language here. This $\sigma_{x}$ is NOT the Young measure associated with $\{a(j x)\}$ but rather with the $Q$-periodic extension of $j x$. We hope this will not cause any confusion.
} 
and

$$
\operatorname{div}\left[\frac{\partial \psi}{\partial \rho}(\nabla u(x))\right]=0 \text { in } Q, \quad u=u_{0} \text { on } \partial \Omega,
$$

the determination of the associated Young measure is a direct consequence of classical corrector results. Notice that if $\nabla u_{j}-U_{j}$ converges strongly to 0 , then the Young measures corresponding to the two sequences $\left\{\left(a(j x), \nabla u_{j}(x)\right)\right\}$ and $\left\{\left(a(j x), U_{j}(x)\right)\right\}$ are the same.

We would like to apply Corollary 3 in this situation. This amounts to finding a constructive way of generating the optimal field in the definition of the integrand for the $\Gamma$-limit, or equivalently, the solution of the cell problem

$$
\operatorname{div}\left[a(\lambda)\left(\rho+\nabla_{\lambda} \xi(\lambda, \rho)\right)\right]=0 .
$$

It is elementary to realize that the dependence of $\rho+\nabla_{\lambda} \xi(\lambda, \rho)$ on $\rho$ is linear so that

$$
\rho+\nabla_{\lambda} \xi(\lambda, \rho)=H(\lambda) \rho .
$$

Therefore, the sequence of fields

$$
U_{j}(x)=H(j x) \nabla u(x)
$$

generates, together with $a_{j}(x)=a(j x)$, the optimal underlying measure for the $\Gamma$-limit. In fact, notice that, by the classical Riemann-Lebesgue lemma,

$$
\lim _{j \rightarrow \infty} \int_{Q} F(x, a(j x), H(j x) \nabla u(x)) d x=\int_{Q} \int_{Q} F(x, \lambda, H(\lambda) \nabla u(x)) d \lambda d x .
$$

By comparing this to (2), we deduce that the joint Young measure for $\left\{\left(a_{j}, U_{j}\right)\right\}$ is the same as the one for $\left\{\left(a_{j}, \nabla u_{j}\right)\right\}$, and as a consequence of Theorem 2, we have

$$
\nabla u_{j}-U_{j} \rightarrow 0 \text { strong in } L^{2}(\Omega) .
$$

As a new example where these ideas can be applied, consider the case of the $p$-laplacian $(p>1)$ still in a periodic setting. Put

$$
I_{j}(v)=\int_{Q} \frac{a(j x)}{p}|\nabla v(x)|^{p} d x, \quad v \in W^{1, p}(Q), Q=(0,1)^{N} .
$$

The integrand for the $\Gamma$-limit, $\psi(\rho)$ ([1]), is given by

$$
\begin{gathered}
\psi(\rho)=\int_{Q} \frac{a(\lambda)}{p}\left|\rho+\nabla_{\lambda} \xi(\lambda, \rho)\right|^{p} d \lambda, \\
\operatorname{div}\left[a(\lambda)\left|\rho+\nabla_{\lambda} \xi(\lambda, \rho)\right|^{p-2}\left(\rho+\nabla_{\lambda} \xi(\lambda, \rho)\right)\right]=0,
\end{gathered}
$$

where $\xi(\lambda, \rho)$ is the $Q$-periodic solution for fixed $\rho$. Therefore, by keeping track of the proof of Theorem 1 in this particular situation (see [11]), we conclude that the field whose existence is guaranteed in Theorem 1 is again of the form

$$
\varphi(\lambda, x)=\nabla u(x)+\nabla_{\lambda} \xi(\lambda, \nabla u(x)) .
$$

The Young measure associated with pairs $\left\{\left(j x, \nabla u_{j}(x)\right)\right\}$ is

$$
\delta_{\nabla u(x)+\nabla_{\lambda} \xi(\lambda, \nabla u(x))} \otimes d \lambda .
$$


In other words,

$$
\lim _{j \rightarrow \infty} \int_{Q} F\left(x, j x, \nabla u_{j}(x)\right) d x=\int_{Q} \int_{Q} F\left(x, \lambda, \nabla u(x)+\nabla_{\lambda} \xi(\lambda, \nabla u(x))\right) d \lambda d x,
$$

provided that $F$ is a Carathéodory integrand, $Q$-periodic in its second variable, for which $\left\{F\left(x, j x, \nabla u_{j}(x)\right)\right\}$ is equiintegrable, and

$$
\lim _{j \rightarrow \infty} \int_{Q} \frac{a(j x)}{p}\left|\nabla u_{j}(x)\right|^{p} d x=\int_{Q} \psi(\nabla u(x)) d x .
$$

Concerning correctors, we can write

$$
H(\lambda, \nabla u(x)) \equiv \varphi(\lambda, x)=\nabla u(x)+\nabla_{\lambda} \xi(\lambda, \nabla u(x)),
$$

where $H(\lambda, \rho)=\rho+\nabla_{\lambda} \xi(\lambda, \rho)$. By directly applying Theorem 2, we conclude that if

$$
U_{j}(x)=H(j x, \nabla u(x)),
$$

then

$$
U_{j}-\nabla u_{j} \rightarrow 0 \text { strong in } L^{p}(\Omega) .
$$

More explicit formulae can be given for a first-order laminate

$$
a(\lambda)=\alpha \chi(\lambda \cdot n)+\beta(1-\chi(\lambda \cdot n))
$$

for specific, positive values $\alpha, \beta$, unit vector $n$, and $\chi=\chi_{t}$, the characteristic function of the interval $(0, t)$ in $(0,1)$ extended by periodicity. In this case, all of the above computations simplify to ([13])

$$
\psi(\rho)=\frac{1}{p} \min _{c \in \mathbf{R}}\left\{t \alpha|\rho+(1-t) c n|^{p}+(1-t) \beta|\rho-t c n|^{p}\right\} .
$$

Let $c(\rho)$ be the optimal solution of this one-dimensional minimization problem so that

$$
\psi(\rho)=\frac{1}{p}\left(t \alpha|\rho+(1-t) c(\rho) n|^{p}+(1-t) \beta|\rho-t c(\rho) n|^{p}\right) .
$$

In this case, the field $\varphi$ is given by

$$
\varphi(\lambda, x)=\nabla u(x)+\left(\chi_{t}(\lambda \cdot n)-t\right) c(\nabla u(x)) n .
$$

If $\left\{u_{j}\right\}$ is such that $u_{j} \rightarrow u$ in $W^{1, p}(Q)$ and

$$
\int_{Q} \frac{\alpha \chi_{t}(j x \cdot n)+\beta\left(1-\chi_{t}(j x \cdot n)\right)}{p}\left|\nabla u_{j}(x)\right|^{p} d x \rightarrow \int_{Q} \psi(\nabla u(x))<+\infty,
$$

then the Young measure corresponding to pairs

$$
\left\{\left(\alpha \chi_{t}(j x \cdot n)+\beta\left(1-\chi_{t}(j x \cdot n)\right), \nabla u_{j}\right)\right\}
$$

is

$$
\delta_{\nabla u(x)+\left(\chi_{t}(\lambda \cdot n)-t\right) c(\nabla u(x)) n} \otimes\left(\chi_{t}(\lambda \cdot n) \delta_{\alpha}+\left(1-\chi_{t}(\lambda \cdot n)\right) \delta_{\beta}\right) d \lambda,
$$

so that

$$
\begin{gathered}
\int_{Q} F\left(x, \alpha \chi_{t}(j x \cdot n)+\beta\left(1-\chi_{t}(j x \cdot n)\right), \nabla u_{j}(x)\right) d x \rightarrow \\
\int_{Q}[t F(x, \alpha, \nabla u(x)+(1-t) c(\nabla u(x)) n)+(1-t) F(x, \beta, \nabla u(x)-t c(\nabla u(x)) n)] d x,
\end{gathered}
$$


provided that $\left\{F\left(x, \alpha \chi_{t}(j x \cdot n)+\beta\left(1-\chi_{t}(j x \cdot n)\right), \nabla u_{j}(x)\right)\right\}$ is equiintegrable.

As before, if we put

$$
H(\lambda, \rho)=\rho+\left(\chi_{t}(\lambda \cdot n)-t\right) c(\rho) n, \quad \varphi(\lambda, x)=H(\nabla u(x), \lambda)
$$

and

$$
U_{j}(x)=H(j x, \nabla u(x))=\nabla u(x)+\left(\chi_{t}(j x \cdot n)-t\right) c(\nabla u(x)) n,
$$

then

$$
U_{j}-\nabla u_{j} \rightarrow 0 \text { strong in } L^{p}(\Omega) .
$$

For the particular case $p=2$, explicit expressions for the mapping $c(\rho)$ can be obtained which lead to corrector results similar to the ones in 3 .

\section{REFERENCES}

[1] Allaire, G. Shape optimization by the homogenization method, 2002, Springer. MR1859696 (2002h:49001)

[2] Balder, E. J. (2000), Lectures on Young measure theory and its applications in economics, in: Proceedings Grado School on Measure Theory and Real Analysis, Rend. Sem. Matem. Trieste 31, suppl. 1, 1-69. MR.1798830 (2001m:49069)

[3] Briane, M., Correctors for the homogenization of a laminate, Adv. Math. Sci. Appl., Gakk. Tokyo, 4, n. 2 (1994), 357-379. MR1294225 (95h:35023)

[4] Cioranescu, D., Donato, P., An Introduction to Homogenization, 1999, Oxford Univ. Press. MR 1765047 (2001j:35019)

[5] Dal Maso, G. (1993), Introduction to $\Gamma$-convergence, Boston MA, Birkhäuser. MR1201152 (94a:49001)

[6] Dal Maso, G., Defranceschi, A., Correctors for the homogenization of monotone operators, Diff. Int. Equations 3 (1990), no. 6, 1151-1166. MR 1073064 (91k:35032)

[7] Kohn, R. V., Strang, G. (1986), Optimal design and relaxation of variational problems, I, II and III, CPAM, 39, 113-137, 139-182 and 353-377. MR820342(87d:49019a)

[8] Murat, F., Tartar, L., H-convergence, in Topics in the mathematical modelling of composite materials, Cherkaev, A., Kohn, R. V., eds., Boston, Birkhäuser, 1997, 21-44. MR1493039

[9] Pankov, A., G-convergence and homogenization of nonlinear partial differential operators, Mathematics and its Applications, 422. Kluwer Academic Publishers, Dordrecht, 1997. MR1482803 (99a:35017)

[10] Pedregal, P. (1997), Parametrized Measures and Variational Principles, Birkhäuser, Basel. MR 1452107 (98e:49001)

[11] Pedregal, P., Г-convergence through Young measures, SIAM J. Math. Anal., 36, n. 2 (2004), 423440. MR2111784 (2005i:49016)

[12] Pedregal, P., Young measures associated with homogenization, SIAM J. Math. Anal., 37, n. 5 (2006), 1454-1464. MR2215272 (2006m:49023)

[13] Serrano, H., Ph.D. Thesis, U. Complutense, 2007.

[14] Valadier M. (1990), Young measures, Methods of Nonconvex Analysis, Lect. Notes in Math., 1446, Springer, 152-188. MR 1079763 (91j:28006) 